\title{
Desigualdades sociais e geográficas no desempenho da assistência pré-natal de uma Região Metropolitana do Brasil
}

\author{
Social and geographical inequalities in the performance of prenatal \\ care in a metropolitan area of Brazil
}

Carolina Dutra Degli Esposti (https://orcid.org/0000-0001-8102-7771) ${ }^{1}$

Edson Theodoro dos Santos-Neto (https://orcid.org/0000-0002-7351-7719) ${ }^{1}$

Adauto Emmerich Oliveira (http://orcid.org/0000-0002-9679-8592) ${ }^{1}$

Claudia Travassos (http://orcid.org/0000-0002-4789-8511) ${ }^{2}$

Rejane Sobrino Pinheiro (http://orcid.org/0000-0002-3361-3626) ${ }^{3}$
${ }^{1}$ Programa de PósGraduação em Saúde Coletiva, Universidade Federal do Espírito Santo. Av. Marechal Campos 1468, Maruípe. 29040-090 Vitória ES Brasil.

carolinaesposti@gmail.com ${ }^{2}$ Instituto de Comunicação e Informação Científica e Tecnológica em Saúde, Fundação Oswaldo Cruz. Rio de Janeiro RJ Brasil. ${ }^{3}$ Instituto de Estudos em Saúde Coletiva, Universidade Federal do Rio de Janeiro. Rio de Janeiro RJ Brasil.

\begin{abstract}
This study analyzed the social and geographical inequalities in the performance of prenatal medical care in the Unified Health System (SUS) in the Metropolitan Region of Grande Vitória, Espírito Santo, Brazil. A cross-sectional study was carried out with 1,209 puerperae living in this region, admitted for childbirth from 2010 to 2011. Data about prenatal care and contextual, enabling, and social characteristics were collected, following the Andersen's Behavioral Model. The performance of prenatal care was classified into five levels, including information on the number of prenatal visits, initial and repetitive examinations, tetanus vaccination, gestational risk management, and participation in educational activities. The likelihood of different levels of prenatal care performance was analyzed using a multivariate multinomial model, according to maternal social variables. High prenatal coverage (98\%) and $4.4 \%$ care adequacy were identified. The likelihood of access to prenatal care was increased by enabling, contextual, and social factors. The relationship between prenatal care quality and pregnant women's social and geographical conditions must be considered in the organization of services to achieve equity and reduce maternal and perinatal morbimortality.
\end{abstract}

Key words Equity in access, Quality of health care, Unified Health System, Prenatal care
Resumo Objetivou-se analisar as desigualdades sociais e geográficas no desempenho da assistência pré-natal no Sistema Único de Saúde, Região Metropolitana da Grande Vitória, Espírito Santo, Brasil. Realizou-se estudo seccional com 1.209 puérperas internadas para o parto em 2010 e 2011. Coletaram-se dados sobre assistência médica prénatal e características contextuais, predisponentes e capacitantes, seguindo o Modelo Comportamental de Andersen. O desempenho da assistência pré-natal foi classificado em cinco niveis incluindo informações sobre: número de consultas pré-natal feitas; realização de exames iniciais e de repetição; vacinação antitetânica; manejo do risco gestacional e participação em atividades educativas. Analisou-se a chance de diferentes níveis de desempenho do pré-natal por meio de modelo multinomial multivariado, segundo variáveis sociais maternas. Verificou-se alta cobertura pré-natal (98\%) e 4,4\% de adequação do cuidado. A chance de acesso ao pré-natal foi aumentada por fatores predisponentes, contextual e capacitantes. A relação entre o pré-natal de qualidade e condições sociais e geográficas da gestante deve orientar a organização dos serviços, visando a equidade e a redução da morbimortalidade materna e perinatal.

Palavras-chave Equidade no acesso, Qualidade da assistência à saúde, Sistema Único de Saúde, Cuidado pré-natal 


\section{Introdução}

O desempenho dos serviços é um importante indicador da estrutura de um sistema de saúde, que deve ser organizado para favorecer a utilização por quem dele necessite. No Brasil, a atenção pré-natal tem sido foco de políticas públicas há várias décadas, sendo importante a análise de seu desempenho, com base na ideia de equidade.

A definição de equidade em saúde, em geral, guarda proximidade com a noção de justiça social, em termos éticos, e de direitos humanos ${ }^{1-4}$. Nesse sentido, a forma como a sociedade se organiza e se desenvolve pode reduzir a oportunidade de algumas pessoas alcançarem um bom nível de saúde, o que caracterizaria uma situação injusta ${ }^{3}$. A Organização Mundial da Saúde (OMS) reconhece que mecanismos sociais, econômicos e políticos estratificam e hierarquizam os indivíduos em grupos sociais que configuram-se como determinantes estruturais das iniquidades em saúde. Subjacentes a esses, os determinantes intermediários incluem as condições de vida, os aspectos psicossociais, os fatores biológicos/ genéticos e comportamentais dos indivíduos e o sistema de saúde. A configuração deste último é o que define o padrão de acesso ao cuidado de saúde dos diferentes grupos sociais e, quando incorpora as diferenças de exposição, de doença e de vulnerabilidade social da população, também por meio de ações intersetoriais, pode minimizar tais diferenciais na vida das pessoas ${ }^{5}$.

Os determinantes sociais interferem na chance individual de obtenção de acesso (utilização) e dos benefícios resultantes dos cuidados em saúde. Nesse sentido, a quinta e atual fase do "Modelo Comportamental da Utilização dos Serviços de Saúde" de Andersen ${ }^{6}$, desenvolvido para a avaliação da utilização aos serviços de saúde, destaca a importância da análise dos determinantes contextuais e individuais no estudo da utilização dos serviços de saúde. O modelo inclui características contextuais capacitantes, tais como a organização e outros fatores relacionados à oferta de serviços, e fatores predisponentes, tais como características demográficas, valores e crenças da comunidade sobre a saúde, medidas de forma agregada. Além desses, destaca fatores individuais e comportamentais de saúde, que incluem os relacionados com os indivíduos, tais como práticas pessoais, que interagem com a utilização e os processos de cuidado e afetam os resultados em saúde e a satisfação dos usuários.

Com relação aos serviços de saúde brasileiros, destaca-se que seu desempenho precisa ser ava- liado, não só com relação ao acesso, mas também à adequação do processo de cuidado de saúde. Dentre os indicadores utilizados, encontram-se elementos relacionados à saúde materno-infantil: percentual de crianças nascidas vivas cujas mães realizaram mais de seis consultas de prénatal; percentual de partos cesáreos em relação ao total de partos; e percentual de gestantes vacinadas contra o tétano ${ }^{7}$. A equidade no desempenho do serviço de pré-natal é uma dimensão transversal que estará presente quando houver igualdade, entre indivíduos e comunidades, de acesso a serviços de saúde adequados às necessidades de saúde, uma vez que, segundo Mooney ${ }^{8}$, a equidade no acesso aos serviços de saúde relaciona-se à noção de justiça. Os determinantes sociais também interferem na chance individual de obtenção de acesso e dos benefícios resultantes dos cuidados de saúde.

Na implementação, no Sistema Único de Saúde (SUS), da atenção primária à saúde, ou atenção básica, a Estratégia Saúde da Família (ESF) teve por objetivo garantir o acesso universal e vem contribuindo para a melhoria do acesso aos serviços de saúde e também para a redução de taxas de importantes agravos, como a mortalidade infantil'? É o principal nível de cuidado responsável pela assistência pré-natal no Brasil. Porém, assim como em outros países ${ }^{10}$, apesar de o número de consultas pré-natal ter aumentado e sua cobertura ser praticamente universal $(98 \%)^{11,12}$, existem baixos percentuais de adequação do cuidado $^{13}$. Análises revelam que a qualidade do cuidado não acompanha a cobertura do serviço de pré-natal e há associação de cuidado menos qualificado com fatores sociais, sendo sua cobertura menor na região Norte, para as indígenas, as mulheres sem companheiro e aquelas com maior número de gestações anteriores ${ }^{12}$. Características como baixa renda, baixa escolaridade, menor idade e ausência de companheiro associam-se a um pré-natal inadequado ${ }^{12,14-17}$, além de haver desigualdades geográficas ${ }^{12,18}$. Essa é uma realidade preocupante, visto que a qualidade do cuidado pré-natal está associada a desfechos perinatais mais favoráveis ${ }^{19-23}$.

Desde 2000, o Programa de Humanização do Parto e Nascimento (PHPN) vem orientando as ações da atenção pré-natal ${ }^{24}$. Porém, como destacaram Victora et al. ${ }^{25}$, a melhoria da qualidade do cuidado costuma ser um processo mais lento do que a ampliação da cobertura. Assim, no Brasil, o acesso e a adequação do cuidado pré-natal ainda constituem grandes desafios mais de duas décadas depois. Entende-se que a análise das desigual- 
dades sociais no acesso ao cuidado pré-natal de qualidade é relevante para a área da saúde públi$\mathrm{ca}$, visto que pode produzir indicativos sobre os determinantes sociais que interferem no acesso aos serviços de saúde de qualidade. Por outro lado, o diagnóstico das desigualdades geográficas fornece indicativos sobre aspectos contextuais que podem facilitar ou dificultar o acesso, permitindo uma compreensão ampliada para tomada de medidas de saúde pública pelos gestores responsáveis pelas políticas de saúde. Dessa forma, a avaliação do desempenho do serviço pré-natal (acesso e adequação), também a partir de fatores contextuais, predisponentes e capacitantes como os apresentados no modelo Comportamental de Andersen ${ }^{6}$, torna-se importante para o planejamento dos serviços.

Diante dessas considerações, este estudo pretende analisar as desigualdades sociais e geográficas no desempenho (utilização e adequação) da assistência pré-natal no Sistema Único de Saúde de uma Região Metropolitana na Região Sudeste do Brasil.

\section{Metodologia}

O Estado do Espírito Santo (ES) localiza-se na Região Sudeste do Brasil e abriga uma população de 3.972 .388 pessoas, aproximadamente $4,9 \% \mathrm{da}$ população total da região. Sua Região Metropolitana da Grande Vitória - ES (RMGV-ES) é formada por sete municípios (Cariacica, Fundão, Guarapari, Serra, Viana, Vila Velha e Vitória), que, juntos, abrigam quase metade da população total do estado $(49,1 \%)^{26}$, produzem aproximadamente $57,7 \%$ do Produto Interno Bruto (PIB) estadual e têm uma taxa de urbanização de $98,2 \%{ }^{27}$.

Entre abril de 2010 e fevereiro de 2011, foram realizados estudos seccionais nessa região, para avaliar a assistência pré-natal do SUS. As descrições detalhadas dos desenhos amostrais foram publicadas ${ }^{28,29}$. O universo populacional foi composto por mulheres que se internaram em um dos nove estabelecimentos de saúde da RMGV-ES que realizavam partos para o SUS no período do estudo. O cálculo da amostra totalizou 1.209 mulheres. Foram excluídas as mulheres que não estavam em posse do cartão da gestante, as que realizaram acompanhamento pré-natal (todo ou parte) no subsistema privado, as que foram acompanhadas em municípios fora da RMGV-ES e as puérperas com menos de 12 horas de realização de parto cesáreo.
O estudo piloto foi realizado entre os meses de março e abril de 2010, com uma amostra de 67 parturientes (não incluídas no estudo), em um dos estabelecimentos onde foi conduzido o estudo principal. As variáveis do estudo foram construídas com base nas informações sobre a assistência médica pré-natal, copiadas do cartão da gestante ou informadas por elas, nas variáveis maternas contidas nos questionários de entrevista e nos indicadores sociais e sobre a organização local dos serviços de saúde dos municípios de residência, divulgados pelo IBGE ${ }^{26}$, ONU/PNUD ${ }^{30}$ e Datasus (Departamento de Informática do SUS. http://www2.datasus.gov.br/SIAB/index. php, acessado em 02/Set/2008).

Para avaliação do desempenho da assistência pré-natal, segundo princípios, atividades e procedimentos do $\mathrm{PHPN}^{24}$, foram verificados no cartão da gestante: número de consultas; início do pré-natal; exames laboratoriais (ABO-Rh, VDRL, urina de rotina (EAS), glicemia de jejum, hemoglobina, hematócrito, Testagem HIV/ AIDS); recebimento de pelo menos uma dose de vacina antitetânica; e repetição dos exames VDRL, EAS e glicemia de jejum. Foram coletadas informações, junto às gestantes, sobre o manejo do risco (classificação e encaminhamento para acompanhamento de gestação de risco) e sua participação em atividades educativas (declaração de recebimento das informações sobre as vantagens da amamentação exclusiva ao peito até os seis meses de vida e sobre a importância da amamentação até os dois anos de idade ou mais (segundo recomendações da $\mathrm{OMS}^{31}$ ) e ter sido incentivada à amamentação em alguma atividade educativa em grupo de gestantes ou em visitas domiciliares $^{28}$.

A interpretação dos achados deu-se a partir da construção de categorias temáticas do Modelo Comportamental de Andersen ${ }^{6}$. As variáveis independentes foram agrupadas em fatores contextuais, predisponentes e capacitantes (sociais). Com relação ao contexto, foram utilizadas variáveis relacionadas à oferta de serviços (cobertura de serviços de atenção básica por tipo de unidade de saúde, segundo informações fornecidas pelas Secretarias Municipais de Saúde); município de moradia, informada pela puérpera durante a entrevista (Vitória ou outro município da RMGV-ES); e o Índice de Desenvolvimento Humano Municipal (IDHM) ${ }^{30}$, atribuídas ao nível da mulher. As variáveis predisponentes foram: idade da mulher (em anos completos) e número de partos anteriores. As variáveis capacitantes (sociais) investigadas foram: escolaridade materna (anos 
completos de estudo); mulher chefe de família; posse de bens de consumo, adaptada a partir do Indice de Bens de Szwarcwald et al. ${ }^{32}$, calculada por (equação 1):

$$
\text { IB }-\sum_{i}\left(1-f_{i}\right) b_{i},(\text { eq. } 1)
$$

na qual $i$ variou de 1 até 8 bens (televisão, geladeira, aparelho de som, telefone fixo, máquina de lavar roupa, automóvel, micro-ondas e microcomputador); $f_{i}$ era a frequência relativa de cada bem; $b i$ era igual a 1 ou zero, respectivamente, na presença ou ausência do bem; e condições de moradia (adequadas - como sendo a existência de condições de moradia internas e externas adequadas; inadequada - condições de moradia internas ou externas inadequadas e altamente inadequada - condições de moradia internas e externas inadequadas). As condições de moradia internas foram classificadas como inadequadas quando a aglomeração humana domiciliar era considerada excessiva (overcrowding) (casos em que a razão entre o número de moradores por cômodo do domicílio, excluindo-se banheiros e cozinha, era maior que 2); ausência de piso revestindo o chão; paredes feitas de argila, palha, madeira, plástico ou metal, e ausência de descarga no banheiro. Foram classificadas como condições de moradia externas inadequadas a ausência de sistema de esgoto ou presença de valão a céu aberto na rua do domicílio ${ }^{33}$. As variáveis maternas foram obtidas a partir de dados da entrevista.

Todos os dados foram digitados no software SPSS versão 17.0 (SPSS Inc. Chicago, Estados Unidos), com exaustivo controle de qualidade e revisão. A variável dependente "desempenho do cuidado pré-natal" foi analisada com cinco categorias: 1 - 5 consultas pré-natal; $\geq 6$ consultas prénatal; $1^{\circ}$ nível de desempenho como sinônimo de acesso, classificado a partir da realização da $1^{\text {a }}$ consulta até a $16^{a}$ semana gestacional e do mínimo seis consultas (número de consultas ajustado pela idade gestacional); e adequação da assistência, analisada como $2^{\circ}$ nível de desempenho, para aquelas que, além do acesso, também realizaram os exames iniciais e a vacinação antitetânica; e como $3^{\circ}$ nível de desempenho para aquelas que, além do acesso, realizaram todos os procedimentos analisados.

Foi realizada a análise estatística de associação bivariada entre o desempenho do cuidado pré-natal e as características maternas (contextuais, predisponentes e capacitantes), pela aplicação dos testes Qui-quadrado e/ou Exato de Fisher, quando havia menos de cinco observa- ções nas células para as variáveis categóricas. Os testes de Mann-Whitney e Kruskall Wallis foram utilizados para a variável posse de bens. A análise das desigualdades geográficas foi realizada pela comparação entre as mulheres residentes em Vitória e as residentes em outros municípios da região, com a finalidade de representar as diferenças entre esses grupos, com relação à organização de serviços de saúde e ao deslocamento que precisa ser realizado para a realização do cuidado em saúde, por exemplo.

A chance de desempenho conforme do cuidado pré-natal foi estimada. Realizou-se análise multinomial multivariada, com o intuito de estimar as associações das variáveis independentes com cada categoria do desfecho, sem estabelecer pressupostos sobre a estrutura de seus coeficientes. Foi elaborado modelo multivariado incluindo as variáveis que apresentaram significância estatística $\alpha<0,20$ na análise bivariada. No modelo final permaneceram apenas as variáveis significantes a um nível de significância $\alpha=0,05$. $O$ intervalo de confiança foi de $95 \%$. Foram incluídas nessa análise somente as gestantes com respostas para todas as variáveis.

O projeto de pesquisa foi aprovado pelo Comitê de Ética em Pesquisa do Centro de Ciências da Saúde da Universidade Federal do Espírito Santo, em 04/11/2009, e pelo Comitê de Ética em Pesquisa da Escola Nacional de Saúde Pública/ Fundação Oswaldo Cruz, em 08/11/2013, atendendo dessa forma às recomendações da Resolução 466/2012, do Conselho Nacional de Saúde. Também foram obtidas as autorizações formais para a execução da pesquisa nos estabelecimentos de saúde (maternidades, hospitais, Secretarias Municipais e Estaduais de Saúde). Todas as puérperas assinaram o Termo de Consentimento Livre e Esclarecido (TCLE).

As análises estatísticas foram realizadas por meio do SPSS versão 17.0 (SPSS Inc. Chicago, Estados Unidos) e do Stata12.

\section{Resultados}

Foram analisados dados de 1.209 puérperas, das quais $360(29,8 \%)$ residiam na capital (Vitória) (Tabela 1). As puérperas não se diferenciaram em relação às características predisponentes "idade" e "número de partos anteriores" nem quanto às características sociais "escolaridade" e "gestante ser chefe de família". Diferenciaram-se com relação à "posse de bens", com residentes em Vitória com maior posse média de bens (médias de 1,40 
Tabela 1. Características maternas e da região de moradia. RMGV-ES, 2010-2011.

\begin{tabular}{|c|c|c|c|c|c|c|c|c|}
\hline & \multirow{3}{*}{ Variáveis } & \multicolumn{7}{|c|}{ Região de Moradia } \\
\hline & & \multicolumn{2}{|c|}{$\begin{array}{c}\text { Total } \\
(n=1209)\end{array}$} & \multicolumn{2}{|c|}{$\begin{array}{c}\text { RMGV-ES } \\
(\mathrm{n}=849)\end{array}$} & \multicolumn{2}{|c|}{$\begin{array}{c}\text { Vitória } \\
(\mathrm{n}=360)\end{array}$} & \multirow[t]{2}{*}{ p-valor ${ }^{\rtimes}$} \\
\hline & & $\mathbf{n}$ & $\%$ & $\mathbf{n}$ & $\%$ & $\mathbf{n}$ & $\%$ & \\
\hline \multirow{7}{*}{ 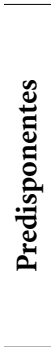 } & Idade $(\mathrm{n}=1209)$ & & & & & & & 0,242 \\
\hline & $\leq 19$ anos & 275 & 22,7 & 185 & 21,8 & 90 & 25,0 & \\
\hline & 20-34 anos & 837 & 69,2 & 600 & 70,7 & 237 & 65,8 & \\
\hline & $\geq 35$ anos & 97 & 8,1 & 64 & 7,5 & 33 & 9,2 & \\
\hline & $\begin{array}{l}\text { Número de partos anteriores } \\
(\mathrm{n}=1209)\end{array}$ & & & & & & & 0,229 \\
\hline & $0-2$ & 1000 & 82,7 & 695 & 81,9 & 305 & 84,7 & \\
\hline & $\geq 3$ & 209 & 17,3 & 154 & 18,1 & 55 & 15,3 & \\
\hline \multirow{11}{*}{ 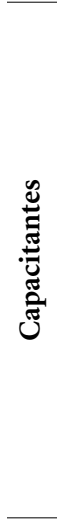 } & Escolaridade $(\mathrm{n}=1199)$ & & & & & & & 0,434 \\
\hline & $\leq 7$ anos & 367 & 30,6 & 266 & 31,7 & 101 & 28,1 & \\
\hline & 8 a 10 anos & 426 & 35,5 & 291 & 34,6 & 135 & 31,7 & \\
\hline & $\geq 11$ anos & 406 & 33,9 & 283 & 33,7 & 123 & 34,3 & \\
\hline & Chefe de família $(\mathrm{n}=1201)$ & & & & & & & 0,893 \\
\hline & Própria gestante & 126 & 10,5 & 88 & 10,4 & 38 & 10,7 & \\
\hline & Outro & 1075 & 89,5 & 757 & 89,6 & 318 & 89,3 & \\
\hline & $\begin{array}{l}\text { Condições gerais de moradia } \\
(\mathrm{n}=1161)\end{array}$ & & & & & & & 0,007 \\
\hline & Altamente inadequadas & 56 & 4,8 & 46 & 5,6 & 10 & 2,9 & \\
\hline & Inadequadas & 306 & 26,4 & 231 & 28,2 & 75 & 22,0 & \\
\hline & Adequadas & 799 & 68,8 & 543 & 66,2 & 256 & 75,1 & \\
\hline \multirow{11}{*}{ 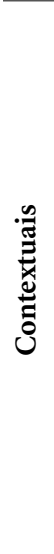 } & $\begin{array}{l}\text { Cobertura de atenção básica } \\
(\mathrm{n}=1205)\end{array}$ & & & & & & & $<0,001$ \\
\hline & UBS & 525 & 43,6 & 476 & 56,3 & 49 & 13,6 & \\
\hline & ESF & 531 & 44,1 & 238 & 28,1 & 293 & 81,6 & \\
\hline & PACS & 149 & 12,4 & 132 & 15,6 & 17 & 4,7 & \\
\hline & $\begin{array}{l}\text { Cobertura de saúde bucal } \\
(\mathrm{n}=1205)\end{array}$ & & & & & & & $<0,001$ \\
\hline & Não & 290 & 24,1 & 284 & 33,6 & 06 & 1,7 & \\
\hline & Sim & 915 & 75,9 & 562 & 66,4 & 353 & 98,3 & \\
\hline & IDHM $(\mathrm{n}=1209)$ & & & & & & & $<0,001$ \\
\hline & Médio & 38 & 3,2 & 38 & 4,5 & - & - & \\
\hline & Alto & 583 & 48,2 & 583 & 68,7 & - & - & \\
\hline & Muito alto & 588 & 48,6 & 228 & 26,9 & 360 & 100,0 & \\
\hline
\end{tabular}

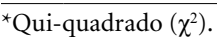

e 1,22 para Vitória e RMGV, respectivamente, $\mathrm{p}$ $=0,009$ ) e às "condições de moradia" (adequação de 75,1\% em Vitória e 66,2\% nos outros municípios da região, $\mathrm{p}=0,007)$. Os contextos diferenciaram. O IDHM de Vitória foi o único classificado como muito alto. A diferença na cobertura de serviços também foi estatisticamente significante: $86,3 \%$ das puérperas de Vitória residiam em áreas cobertas por ESF e PACS e 98,3\% por serviços odontológicos, enquanto essas cober- turas eram de apenas $43,7 \%$ e $66,4 \%$ nos outros municípios da região, respectivamente $(\mathrm{p}<0,001$ para ambos).

As prevalências de realização de cada exame inicial foram superiores a $61 \%$ em ambas as regiões, porém foram maiores no município de Vitória (Tabela 2), estatisticamente superiores para os exames de tipagem sanguínea (ABO-Rh) e o primeiro VDRL. Destaca-se que a prevalência de cumprimento de pelo menos uma dose da vacina 
Tabela 2. Distribuição dos procedimentos da assistência pré-natal, segundo região de moradia. RMGV-ES, 20102011.

\begin{tabular}{|c|c|c|c|c|c|c|c|}
\hline \multirow{2}{*}{ Atividades do PHPN } & \multicolumn{2}{|c|}{ Total } & \multicolumn{2}{|c|}{ RMGV-ES } & \multicolumn{2}{|c|}{ Vitória } & \multirow[t]{2}{*}{ p-valor } \\
\hline & $\mathbf{n} \dagger$ & $\%$ & $\mathbf{n}$ & $\%$ & $\mathbf{n}$ & $\%$ & \\
\hline Procedimentos do PHPN & 1183 & 100,0 & 830 & 100,0 & 353 & 100,0 & \\
\hline Início do pré-natal até o $4^{\circ}$ mês & 600 & 55,7 & 404 & 52,6 & 196 & 63,4 & 0,001 \\
\hline Consultas de pré-natal & & & & & & & $<0,001$ \\
\hline $1-5$ & 425 & 35,9 & 349 & 42,0 & 76 & 21,5 & \\
\hline $6+$ & 758 & 64,1 & 481 & 58,0 & 277 & 78,5 & \\
\hline Tipagem sanguínea (ABO-Rh) & 762 & 64,4 & 511 & 61,6 & 251 & 71,1 & 0,002 \\
\hline Primeiro VDRL & 864 & 73,0 & 590 & 71,1 & 274 & 77,6 & 0,020 \\
\hline Primeiro EAS & 795 & 67,2 & 558 & 67,2 & 237 & 67,1 & 0,976 \\
\hline Primeira glicemia & 861 & 72,8 & 596 & 71,8 & 265 & 75,1 & 0,249 \\
\hline Hemoglobina & 844 & 71,3 & 585 & 70,5 & 259 & 73,4 & 0,315 \\
\hline Hematócrito & 770 & 65,1 & 534 & 64,3 & 236 & 66,9 & 0,406 \\
\hline Anti-HIV & 776 & 64,8 & 545 & 65,7 & 221 & 62,6 & 0,314 \\
\hline Imunização antitetânica & 427 & 36,1 & 304 & 36,6 & 123 & 34,8 & 0,559 \\
\hline Repetição VDRL & 397 & 33,6 & 263 & 31,7 & 134 & 38,0 & 0,037 \\
\hline Repetição EAS & 403 & 34,1 & 264 & 31,8 & 139 & 39,4 & 0,012 \\
\hline Repetição glicemia & 427 & 36,1 & 278 & 33,5 & 149 & 42,2 & 0,004 \\
\hline Manejo do risco gestacional & 1099 & 93,3 & 769 & 92,9 & 330 & 94,3 & 0,376 \\
\hline Atividades educativas & 215 & 18,2 & 145 & 17,5 & 70 & 19,9 & 0,325 \\
\hline Níveis de Desempenho $(n=1072)$ & 1072 & 100,0 & 766 & 100,0 & 306 & 100,0 & $<0,001$ \\
\hline $1-5$ consultas & 389 & 36,3 & 323 & 42,2 & 66 & 21,6 & \\
\hline$\geq 6$ consultas & 173 & 16,1 & 111 & 14,5 & 62 & 20,3 & \\
\hline $1^{\circ}$ nível & 411 & 38,3 & 259 & 33,8 & 152 & 49,7 & \\
\hline $2^{\circ}$ nível & 46 & 4,3 & 35 & 4,6 & 11 & 3,6 & \\
\hline 3o nível & 53 & 4,9 & 38 & 5,0 & 15 & 4,9 & \\
\hline
\end{tabular}

* Teste qui-quadrado $\left(\chi^{2}\right)$ † Na análise descritiva foram incluídas todas as mulheres com informação, em cada uma das variáveis, enquanto que na regressão foram incluídas somente aquelas com informação disponível para o conjunto das variáveis.

antitetânica foi inferior a 37\% em ambas as regiões do estudo, sem significância estatística para a diferença.

As prevalências dos exames de repetição foram inferiores a $43 \%$, tendo sido estatisticamente superiores para as residentes no município de Vitória. O manejo do risco gestacional ocorreu para 93,3\% das gestantes deste estudo, enquanto que as atividades educativas foram cumpridas por menos de $20 \%$ delas, ambos os procedimentos sem diferenças estatisticamente significativas entre as regiões (Tabela 2).

Ainda segundo informações da Tabela 2, considerando-se as 1.072 mulheres com informações para a análise do desempenho do pré-natal, e baseando-se no cumprimento total de procedimentos recomendados pelo PHPN, observa-se que, de um modo geral, Vitória apresentou padrão estatisticamente superior ao conjunto dos outros municípios da região $(\mathrm{p}<0,001)$. Ainda assim, das gestantes residentes em Vitória, apenas 62 $(20,3 \%)$ realizaram seis ou mais consultas prénatal e apenas $152(49,7 \%)$ tiveram o acesso adequado ao pré-natal ( $1^{\circ}$ nível). Das residentes nos demais municípios da RMGV-ES, 323 (42,2\%) realizaram de uma a cinco consultas e apenas 259 $(33,8 \%)$ tiveram acesso adequado ao pré-natal ( $1^{\circ}$ nível). A análise dos $2^{\circ}$ e $3^{\circ}$ níveis de desempenho, que incluem procedimentos do processo de cuidado pré-natal, apresentou uma queda nas prevalências de adequação, na medida em que o número de procedimentos preconizados aumentou, com $4,3 \%$ das gestantes recebendo todos os cuidados incluídos no $2^{\circ}$ nível de adequação, en- 
quanto $4,9 \%$ receberam todos os procedimentos previstos no PHPN, respectivamente, para o conjunto das mulheres estudadas. O cumprimento do total de procedimentos recomendados pelo PHPN foi semelhante nas duas regiões (5\%).

A análise bivariada, apresentada na Tabela 3, revelou marcadas desigualdades no desempenho do pré-natal. O nível de desempenho foi associado com maior idade $(\mathrm{p}=0,040)$, menor número de partos $(\mathrm{p}=0,001)$, maior escolaridade ( $\mathrm{p}$
$=0,002)$, a gestante ser a chefe da família $(\mathrm{p}=$ 0004), cobertura de atenção básica por UBS ( $\mathrm{p}=$ $0,005)$ e residir nos demais municípios da RMGV-ES $(\mathrm{p}<0,001)$.

$\mathrm{Na}$ análise multivariada, a chance de cumprimento de cada nível de desempenho foi diferente entre as variáveis analisadas (Tabela 4). Com relação às variáveis predisponentes, quanto maior a idade, maior foi a chance de uma puérpera cumprir o $1^{\circ}$ nível (acesso) e o $3^{\circ}$ nível de

Tabela 3. Desempenho da assistência pré-natal, segundo características do contexto, predisponentes e capacitantes. RMGV-ES, 2010-2011.

\begin{tabular}{|c|c|c|c|c|c|c|c|c|c|c|c|c|c|c|}
\hline & \multirow[t]{2}{*}{ Variáveis } & \multicolumn{2}{|c|}{$\begin{array}{c}\text { Total } \\
(\mathrm{n}=1013)\end{array}$} & \multicolumn{2}{|c|}{$\begin{array}{l}1-5 \text { cons. } \\
(n=365)\end{array}$} & \multicolumn{2}{|c|}{$\begin{array}{l}\geq 6 \text { cons. } \\
(n=165)\end{array}$} & \multicolumn{2}{|c|}{$\begin{array}{c}1^{\circ} \text { nível } \\
(\mathrm{n}=390)\end{array}$} & \multicolumn{2}{|c|}{$\begin{array}{l}2^{\circ} \text { nível } \\
(\mathrm{n}=42)\end{array}$} & \multicolumn{2}{|c|}{$\begin{array}{l}33^{\circ} \text { nível } \\
(\mathbf{n}=51)\end{array}$} & \multirow[t]{2}{*}{ p-valor ${ }^{\star}$} \\
\hline & & $\mathbf{n}$ & $\%$ & $\mathbf{n}$ & $\%$ & $\mathbf{n}$ & $\%$ & $\mathbf{n}$ & $\%$ & $\mathbf{n}$ & $\%$ & $\mathbf{n}$ & $\%$ & \\
\hline \multirow{7}{*}{ 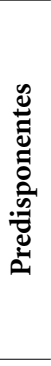 } & Idade & & & & & & & & & & & & & 0,040 \\
\hline & $\leq 19$ anos & 224 & 22,1 & 94 & 42,0 & 41 & 18,3 & 76 & 33,9 & 08 & 3,6 & 05 & 2,2 & \\
\hline & 20-34 anos & 706 & 69,7 & 245 & 34,7 & 108 & 15,3 & 280 & 39,7 & 34 & 4,8 & 39 & 5,5 & \\
\hline & $\geq 35$ anos & 83 & 8,2 & 26 & 31,3 & 16 & 19,3 & 34 & 41,0 & 00 & 0,0 & 07 & 8,4 & \\
\hline & $\begin{array}{l}\text { Número de partos } \\
\text { anteriores }\end{array}$ & & & & & & & & & & & & & 0,001 \\
\hline & $0-2$ & 843 & 83,2 & 282 & 33,5 & 135 & 16,0 & 343 & 40,7 & 38 & 4,5 & 45 & 5,3 & \\
\hline & $\geq 3$ & 170 & 16,8 & 83 & 48,8 & 30 & 17,6 & 47 & 12,1 & 04 & 2,4 & 06 & 3,5 & \\
\hline \multirow{11}{*}{ 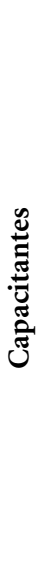 } & Escolaridade & & & & & & & & & & & & & 0,002 \\
\hline & $\leq 7$ anos & 302 & 29,8 & 128 & 42,4 & 54 & 17,9 & 98 & 32,5 & 08 & 2,6 & 14 & 4,6 & \\
\hline & 8 a 10 anos & 352 & 34,7 & 139 & 39,5 & 47 & 13,4 & 132 & 37,5 & 17 & 4,8 & 17 & 4,8 & \\
\hline & $\geq 11$ anos & 359 & 35,4 & 98 & 27,3 & 64 & 17,8 & 160 & 44,6 & 17 & 4,7 & 20 & 5,6 & \\
\hline & Chefe de família & & & & & & & & & & & & & 0,004 \\
\hline & Própria gestante & 112 & 11,1 & 50 & 44,6 & 20 & 17,9 & 29 & 25,9 & 02 & 1,8 & 11 & 9,8 & \\
\hline & Outro & 901 & 88,9 & 315 & 35,0 & 145 & 16,1 & 361 & 40,1 & 40 & 4,4 & 40 & 4,4 & \\
\hline & $\begin{array}{l}\text { Condições gerais de } \\
\text { moradia }\end{array}$ & & & & & & & & & & & & & 0,060 \\
\hline & $\begin{array}{l}\text { Altamente } \\
\text { inadequadas }\end{array}$ & 48 & 4,7 & 23 & 47,9 & 07 & 14,6 & 12 & 25,0 & 03 & 6,3 & 03 & 6,3 & \\
\hline & Inadequadas & 253 & 25,0 & 99 & 39,1 & 52 & 20,6 & 81 & 32,0 & 10 & 4,0 & 11 & 4,3 & \\
\hline & Adequadas & 712 & 70,3 & 243 & 34,1 & 106 & 14,9 & 297 & 41,7 & 29 & 4,1 & 37 & 5,2 & \\
\hline \multirow{11}{*}{ 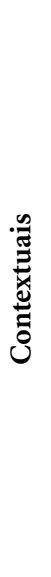 } & $\begin{array}{l}\text { Cobertura de } \\
\text { atenção básica }\end{array}$ & & & & & & & & & & & & & 0,005 \\
\hline & UBS & 452 & 44,6 & 184 & 40,7 & 58 & 12,8 & 160 & 35,4 & 24 & 5,3 & 26 & 5,8 & \\
\hline & ESF & 446 & 44,0 & 135 & 30,3 & 89 & 20,0 & 185 & 41,5 & 17 & 3,8 & 20 & 4,5 & \\
\hline & PACS & 115 & 11,4 & 46 & 40,0 & 18 & 15,7 & 45 & 39,1 & 01 & 0,9 & 05 & 4,3 & \\
\hline & $\begin{array}{l}\text { Município de } \\
\text { moradia }\end{array}$ & & & & & & & & & & & & & $<0,001$ \\
\hline & RMGV-ES & 729 & 72,0 & 302 & 41,5 & 105 & 14,4 & 251 & 34,4 & 33 & 4,5 & 38 & 5,2 & \\
\hline & Vitória & 284 & 28,0 & 63 & 22,2 & 60 & 21,1 & 139 & 48,9 & 09 & 3,2 & 13 & 4,6 & \\
\hline & IDHM & & & & & & & & & & & & & 0,127 \\
\hline & Médio & 29 & 2,9 & 11 & 37,9 & 03 & 10,3 & 13 & 44,8 & 02 & 6,9 & 00 & 0,0 & \\
\hline & Alto & 493 & 48,7 & 197 & 40,0 & 75 & 15,2 & 171 & 34,7 & 22 & 4,5 & 28 & 5,7 & \\
\hline & Muito alto & 491 & 48,5 & 157 & 32,0 & 87 & 17,7 & 206 & 42,0 & 18 & 3,7 & 23 & 4,7 & \\
\hline
\end{tabular}


Tabela 4. Modelo final da razão de chance (IC) dos níveis de desempenho da assistência pré-natal. RMGV-ES, 2010-2011.

\begin{tabular}{|c|c|c|c|c|c|c|c|c|c|}
\hline & \multirow{2}{*}{ Variáveis } & \multicolumn{2}{|c|}{$\begin{array}{l}\geq 6 \text { ou mais } \\
\text { consultas }\end{array}$} & \multicolumn{2}{|c|}{$1^{\circ}$ nível } & \multicolumn{2}{|c|}{$2^{\circ}$ nível } & \multicolumn{2}{|c|}{$3^{\circ}$ nível } \\
\hline & & $\begin{array}{l}\text { Odds } \\
\text { Ratio }\end{array}$ & IC95\% & $\begin{array}{l}\text { Odds } \\
\text { Ratio }\end{array}$ & IC95\% & $\begin{array}{l}\text { Odds } \\
\text { Ratio }\end{array}$ & IC95\% & $\begin{array}{l}\text { Odds } \\
\text { Ratio }\end{array}$ & IC95\% \\
\hline \multirow{7}{*}{ 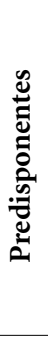 } & Idade & & & & & & & & \\
\hline & $\leq 19$ anos & 1,00 & - & 1,00 & - & 1,00 & - & 1,00 & - \\
\hline & 20-34 anos & 1,00 & $0,62-1,60$ & $1,53^{*}$ & $1,05-2,24$ & 1,71 & $0,73-4,01$ & $2,71^{*}$ & $1,15-6,41$ \\
\hline & $\geq 35 \operatorname{anos}^{* *}$ & 1,61 & $0,71-3,63$ & $2,56^{*}$ & $1,29-5,06$ & - & - & $7,72^{*}$ & $2,38-25,1$ \\
\hline & $\begin{array}{l}\text { Número de partos } \\
\text { anteriores }\end{array}$ & & & & & & & & \\
\hline & $0-2$ & 1,00 & - & 1,00 & - & 1,00 & - & 1,00 & - \\
\hline & $\geq 3$ & 0,73 & $0,43-1,26$ & $0,45^{*}$ & $0,29-0,71$ & 0,51 & $0,17-1,58$ & $0,27^{*}$ & $0,11-0,67$ \\
\hline \multirow{7}{*}{ 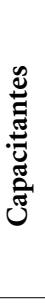 } & Escolaridade & & & & & & & & \\
\hline & $\leq 7$ anos & 1,00 & - & 1,00 & - & 1,00 & - & 1,00 & - \\
\hline & 8 a 10 anos & 0,74 & $0,46-1,19$ & 1,04 & $0,71-1,51$ & 1,59 & $0,65-3,90$ & 1,02 & $0,50-2,11$ \\
\hline & $\geq 11$ anos & 1,52 & $0,94-2,47$ & $1,68^{*}$ & $1,13-2,50$ & 1,99 & $0,78-5,06$ & 1,52 & $0,74-3,11$ \\
\hline & Chefe de família & & & & & & & & \\
\hline & Outro & 1,00 & - & 1,00 & - & 1,00 & - & 1,00 & - \\
\hline & Própria gestante & 1,23 & $0,69-2,19$ & $1,92^{*}$ & $1,15-3,22$ & 2,72 & $0,62-11,8$ & 0,66 & $0,32-1,37$ \\
\hline \multirow{7}{*}{ 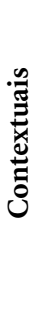 } & $\begin{array}{l}\text { Cobertura da atença } \\
\text { básica }\end{array}$ & & & & & & & & \\
\hline & UBS & 1,00 & - & 1,00 & - & 1,00 & - & 1,00 & - \\
\hline & ESF & $1,59^{*}$ & $1,01-2,48$ & 1,17 & $0,82-1,67$ & 0,99 & $0,47-2,09$ & 0,84 & $0,43-1,60$ \\
\hline & PACS & 1,30 & $0,70-2,44$ & 1,20 & $0,74-1,94$ & 0,17 & $0,22-1,27$ & 0,86 & $0,33-2,20$ \\
\hline & Município de mora & & & & & & & & \\
\hline & RMGV-ES & 1,00 & - & 1,00 & - & 1,00 & - & 1,00 & - \\
\hline & Vitória & $2,28^{*}$ & $1,43-3,64$ & $2,52^{*}$ & $1,70-3,73$ & 1,23 & $0,51-2,98$ & $2,27^{*}$ & $1,13-4,55$ \\
\hline
\end{tabular}

${ }^{\star}$ p-valor $<0,05$. ${ }^{*}$ não havia observações para idade $\geq 35$ anos no grupo que cumpriu o $2^{\circ}$ nível de desempenho. Dessa forma, $o$ Odds Ratio e o intervalo de confiança não puderam ser estimados.

desempenho, em relação à chance de realizar menos de seis consultas. O maior número de partos anteriores esteve relacionado com uma menor chance de adequação: $55 \%$ menor para a chance de cumprir o $1^{\circ}$ nível e $73 \%$ menor de cumprir o $3^{\circ}$ nível.

A desigualdade social foi observada no acesso ao pré-natal ( $1^{\circ}$ nível), em comparação a realizar menos de seis consultas, não sendo estatisticamente significante a diferença encontrada para o cumprimento dos $2^{\circ}$ e $3^{\circ}$ níveis (Tabela 4 ). A chance de uma puérpera iniciar o pré-natal até o $4^{\circ}$ mês e realizar seis ou mais consultas ( $1^{\circ}$ nível de desempenho), comparada à chance de realizar de uma a cinco consultas foi $68 \%$ maior para aquelas com 11 ou mais anos de escolaridade e $92 \%$ maior se ela fosse a chefe de família. A puérpera não ser a chefe da família foi associado com o melhor nível de desempenho. Também foram observadas desigualdades contextuais no acesso e no processo de cuidado: morar em área com cobertura de ESF associou-se ao aumento de 59\% na chance de uma puérpera realizar seis ou mais consultas, comparada à chance de realizar menos de seis consultas, porém, não se relacionou à chance de receber pré-natal de qualidade $\left(1^{\circ}\right.$ a $3^{\circ}$ níveis de desempenho). Morar em Vitória aumentou em torno de duas vezes a chance de a gestante realizar seis ou mais consultas, ter acesso adequado ( $1^{\circ}$ nível de desempenho) ou um cuidado pré-natal de qualidade ( $3^{\circ}$ nível de desempenho), comparada à chance de realizar de uma a cinco consultas pré-natal.

\section{Discussão}

Este trabalho mostrou que, nos municípios da RMGV-ES, embora a cobertura pré-natal tenha alcançado $98 \%$, somente cinco em cada cem 
puérperas atendidas no SUS o realizaram de forma totalmente adequada. $\mathrm{O}$ processo do cuidado teve uma baixa adequação, com um pequeno percentual de gestantes que realizaram todos os procedimentos previstos no PHPN (3o nível de desempenho) (5\%). Mostrou, ainda, que existem desigualdades sociais e geográficas no acesso a esse serviço, com grande diferença nos níveis de desempenho alcançados. Do ponto de vista do cuidado integral à saúde materno-infantil, o prénatal deveria ser organizado de forma a garantir uma assistência adequada para as gestantes, independentemente de suas características sociais e geográficas ${ }^{34}$.

Esse resultado foi semelhante ao observado em outros estudos, que mostraram uma cobertura pré-natal de aproximadamente $99 \%$ para o Brasil $^{11,12}$ e baixos percentuais de adequação do cuidado, independente da fonte da informação e dos critérios de classificação ${ }^{13,35}$. Ao interpretar os resultados é preciso considerar que quanto mais exigente o critério, maior a prevalência de inadequação ${ }^{35}$. Destaca-se que o critério utilizado no presente estudo visou contemplar os procedimentos indicados no PHPN em sua amplitude e que o baixo percentual ao $3^{\circ}$ nível de desempenho representa a realização de todos os procedimentos analisados previstos para o pré-natal ${ }^{24}$. Diferente do aqui proposto, a maior parte dos estudos sobre a temática foca apenas no acesso (medido pelo número de consultas e momento do início do pré-natal) e/ou em uma lista mais reduzida de procedimentos ${ }^{13-17,35}$.

É preciso discutir e evidenciar que o processo de implementação de uma política depende de vários fatores, como da existência de recursos financeiros suficientes e de quantidade e qualidade de recursos humanos para atender à universalidade, por exemplo. Por isso, é preciso avaliar não só a cobertura dos programas, mas também a qualidade dos serviços prestados. Entende-se, porém, que a ampliação da cobertura pode ser atingida num prazo mais curto do que a melhoria da qualidade do cuidado, que costuma ser um processo mais lento e complexo ${ }^{25}$. Ademais, os estudos de avaliação do cuidado pré-natal, em geral, focam na proporção de gestantes que realizaram o mínimo de seis consultas recomendadas pelo Ministério da Saúde. Nem mesmo o PHPN tem sido utilizado, exceto pelo próprio Ministério $^{35}$.

O desempenho da assistência pré-natal não foi o mesmo em relação às duas regiões estudadas e aos fatores analisados, organizados segundo contextuais, predisponentes e capacitantes, que, de acordo com Andersen ${ }^{6}$, afetam a utilização dos serviços de saúde. Estudos brasileiros têm analisado a relação entre o cuidado pré-natal e as características sociais desfavoráveis das gestantes ${ }^{12,14,16,17,36,37}$. Assim, também vários países pelo mundo ${ }^{10}$.

Barreiras de acesso geográfico, organizacionais, indisponibilidade de recursos materiais e humanos, custos diretos e indiretos para a obtenção do cuidado, expectativas não atendidas sobre procedimentos e aspectos subjetivos do cuidado apresentam-se às gestantes, e requerem uma capacidade de enfrentamento por parte delas ${ }^{38}$. Outros estudos também revelaram aspectos implicados no acesso, principalmente relacionados à aceitabilidade e à sua relação com a adesão ao cuidado, ao protagonismo das usuárias na construção dos itinerários terapêuticos e à percepção sobre a oferta de recursos materiais e huma$\operatorname{nos}^{37,39-42}$.

Apesar de Vitória ter apresentado melhor desempenho no acesso ao pré-natal, as prevalências de adequação às etapas do processo de cuidado foram semelhantes ao conjunto dos demais municípios da RMGV-ES. Observou-se uma queda na prevalência na comparação entre o $1^{\circ}$ (acesso) e o 30 (adequação total) níveis de desempenho. A baixa adequação do cuidado verificada pode estar relacionada a problemas no processo de cuidado, como o desconhecimento ou a falta de adesão aos protocolos e a falta de registro de informações no cartão da gestante (utilizado como fonte de dados para o parto e também em pesquisas científicas). Além disso, outros fatores podem estar relacionados, como problemas na organização dos serviços, que acabam por criar barreiras para a realização dos procedimentos preconizados pelo PHPN de forma fácil e conveniente: sistemas de marcação deficientes; grande distância dos locais onde os exames são realizados; demora na liberação dos resultados; e barreiras relacionadas à disponibilidade de laboratórios de análises clínicas, como citado por Thiede et al. ${ }^{34}$.

Esses resultados seguiram, no entanto, os índices nacionais, pois a baixa adequação do processo de cuidado também foi observada em várias regiões brasileiras ${ }^{13,14,28,35,43-50}$ e no exterior ${ }^{10}$. Tudo isso envolve, contudo, a necessidade de realocação e, em muitos casos, o maior aporte de recursos financeiros para esses serviços de saúde, além da necessária capacidade gerencial para o planejamento e a avaliação das ações, de treinamento e de adesão da equipe de saúde. A implementação do PHPN é mais complexa do que parece ao primeiro olhar e depende dos esforços 
dos diversos atores sociais e fatores (dependentes de recursos) envolvidos.

Outro importante resultado que merece destaque foi a baixa prevalência de recebimento de informações importantes pelas gestantes. Apesar da reconhecida importância da educação em saúde para esse grupo ${ }^{50}$, parece haver uma lacuna a respeito das atividades educativas referentes à assistência pré-natal na atenção primária à saúde ${ }^{51}$. As atividades educativas deveriam receber maior atenção quando do planejamento e da execução do processo de assistência pré-natal, pois poderiam diminuir a assimetria entre o conhecimento das gestantes e as prescrições do serviço ${ }^{52,53}$. A informação é fator necessário para que os indivíduos possam fazer escolhas quanto à utilização de um serviço de saúde e para o processo de cuidado e, sendo assim, é um importante determinante da qualidade do sistema de saúde e afeta o acesso aos cuidados de forma expressiva ${ }^{34}$.

Considerando o Modelo Comportamental da Utilização dos Serviços de Saúde de Andersen ${ }^{6}$, quanto às desigualdades sociais no desempenho do pré-natal, observou-se que os diversos fatores capacitantes analisados não se comportaram de maneira uniforme para cada um dos níveis de desempenho do pré-natal. A maior escolaridade da mulher e o fato dela ser a chefe da família agiram aumentando a chance de acesso ao pré-natal ( $1^{\circ}$ nível), porém nenhum fator social apresentou influência sobre o processo de cuidado $\left(2^{\circ} \mathrm{e}\right.$ $3^{\circ}$ níveis de desempenho).

As desigualdades sociais no acesso ao pré-natal de qualidade estão discutidas na literatura. De maneira geral, os estudos têm demonstrado que características como baixa renda, baixa escolaridade, menor idade e ausência de companheiro estão associadas ao recebimento de uma assistência pré-natal menos adequada no Brasil $12,14-17,36 \mathrm{e}$ em outros países em desenvolvimento ${ }^{10,54}$.

Ainda considerando o mesmo modelo ${ }^{6} \mathrm{com}$ relação aos fatores predisponentes, no presente estudo, o maior número de partos anteriores diminuiu a chance de acesso ao pré-natal ( $1^{\circ}$ nível) e de receber um cuidado de qualidade ( $3^{\circ}$ nível), o que pode estar relacionado à influência de uma experiência passada negativa com relação ao cuidado pré-natal ou que tenha dado às gestantes a sensação de já terem o conhecimento necessário e maior autonomia na decisão de realizar ou não determinado procedimento do cuidado. Segundo estudo de Figueroa et al. ${ }^{53}$, para gestantes que tiveram uma experiência prévia de cuidado pré-natal ruim, o melhor conhecimento sobre o cuidado pré-natal e os sintomas da gravi- dez implicam numa atitude positiva para a busca pelo cuidado pré-natal, apesar de reconhecerem a complexa relação entre conhecimento, apoio recebido de sistemas de seguridade social e atitudes. Já o estudo de Viellas et al. ${ }^{12}$ mostrou que, no Brasil, para as gestantes com maior número de partos, um dos motivos para o início mais tardio do pré-natal foram as situações de gestações não desejadas, que influenciaram também na não realização do pré-natal das gestantes sem companheiro. Além disso, como cuidadoras, as mulheres com um maior número de partos anteriores também dividem o tempo com os filhos, dedicando menos tempo a si próprias.

Por outro lado, a maior idade e a compreensão sobre a necessidade do cuidado pré-natal e sobre o risco de não receber esse acompanhamento podem ser explicação para a ocorrência de maiores chances para o acesso ao pré-natal $\left(1^{\circ}\right.$ nível) ou para a realização de todas as etapas do cuidado ( $3^{\circ}$ nível), independentemente da maior paridade. Estudos demonstram a associação entre menor idade materna e início tardio do prénatal ${ }^{12,55}$, menor prevalência de realização de seis ou mais consultas ${ }^{12,55,56} \mathrm{e}$ a maior chance de inadequação do pré-natal ${ }^{35,57,58}$.

Quanto às desigualdades capacitantes (sociais), a escolaridade mais elevada, ajustada pelas demais variáveis do modelo, esteve associada a maior chance de acesso realizado ( $1^{\circ}$ nível de desempenho), porém não modificou a chance de cumprimento do maior nível de desempenho pré-natal ( $3^{\circ}$ nível), o que demonstra que esse fator social não influenciou no cumprimento do cuidado pré-natal como um todo. A maior escolaridade costuma estar associada ao início precoce do pré-natal e ao cumprimento do mínimo de seis consultas ${ }^{12,56}$. Porém, diferentemente do aqui observado, estudos constatam que a maior escolaridade também costuma mostrar associação ao cumprimento dos exames indicados para cada período pré-natal ${ }^{56}$ e ao atendimento de todas as etapas do cuidado proposto ${ }^{56,58}$. É importante apontar que comportamentos de vida mais saudáveis estão associados à maior escolaridade ${ }^{59}$.

Outro fator capacitante analisado, a própria gestante ser a chefe de família, relacionou-se positivamente ao acesso ao pré-natal, não influenciando no processo de cuidado. Assim também, estudos realizados com gestantes usuárias do SUS nos municípios de Niterói-RJ e do Rio de Janeiro-RJ não mostraram associação entre a gestante ser a chefe de família e um pré-natal adequado $^{60}$. Entretanto, essa é uma variável relacionada à vulnerabilidade e à exclusão social, que 
coloca as mulheres diante de dificuldades sociais, econômicas e de violência, configurando um quadro de baixa autoestima, frustrações, medos e anseios e, ao mesmo tempo, de coragem e luta ${ }^{61}$. Pode-se inferir que a autonomia dessas mulheres atua como potencializadora do acesso ao prénatal, que possui alta prevalência na população em geral, enquanto a qualificação do processo de cuidado, que possui baixa frequência, não sofreu influência dos fatores sociais.

Os resultados também mostraram que, como esperado, maiores barreiras de acesso ocorrem em contextos menos desenvolvidos. Essa afirmativa é reforçada pela identificação de que melhores resultados em quase todos os níveis de desempenho foram observados para as residentes em Vitória. É importante a análise dos fatores relacionados às desigualdades geográficas. Esse município é a capital do Estado, concentra aproximadamente $50 \%$ do PIB da RMGV-ES e recebe $21,5 \%$ do Imposto sobre Operações relativas à Circulação de Mercadorias e Prestação de Serviços de Transporte Interestadual e Intermunicipal e de Comunicação (ICMS) estadual. A despesa per capita com saúde em Vitória, no ano de 2011, foi o dobro da média do gasto dos demais municípios da região $0^{62}$ e, tendo esse município um sistema de saúde melhor organizado do que os dos demais da região $0^{62,63}$, pressupõe-se, então, que apresente menos barreiras geográficas e organizacionais para o acesso aos serviços.

A maior chance de acesso ao pré-natal em Vitória (fator contextual) pode estar relacionada ao potencial de captação da ESF, que tem uma alta cobertura nesse município ${ }^{63}$. Além disso, é preciso refletir que Vitória apresenta $100 \%$ de população urbana, uma extensão territorial de apenas $98 \mathrm{~km}^{2}$ e serviços de saúde bem organizados. Apesar dos demais municípios da RMGV-ES não apresentarem um alto percentual de população rural, variando de $0,2 \%$ a $15,6 \%$ da população residente, as extensões territoriais desses municípios são maiores (entre $210 \mathrm{~km}^{2}$ e $594 \mathrm{~km}^{2}$ ) (http://cidades.ibge.gov.br/xtras/home.php) e seus serviços de saúde são menos organizados ${ }^{63}$. Essa realidade pode criar barreiras geográficas à continuidade do processo de cuidado e influenciar em sua adequação, pelas maiores distâncias a serem percorridas pelas gestantes residentes nos outros municípios da RMGV-ES, quando comparadas às distâncias percorridas pelas gestantes residentes em Vitória.

Esses fatores estão relacionados ao conceito de disponibilidade de serviços ${ }^{34}$. Quando a distribuição geográfica dos serviços de saúde não tem como referencial as necessidades de saúde dos residentes de uma região, desigualdades geográficas no acesso aos serviços de saúde de qualidade ocorrem $^{64}$. Como afirma Mooney ${ }^{8}$, a equidade no acesso também pode ser entendida como a aplicação dos recursos de forma proporcional às necessidades da população.

O processo de cuidado pré-natal não mostrou ser influenciado pelo fator contextual do tipo de modelo de Atenção Primária à Saúde (UBS, PACS e ESF). A adequação envolve fatores que vão além da disponibilidade do serviço. Pode ser influenciado pelo comportamento e conhecimento dos profissionais de saúde, por sistemas de marcação eficientes, pela disponibilidade e acessibilidade (acesso geográfico) a serviços de realização de exames complementares e pela adesão das gestantes aos procedimentos prescritos, dentre outros. Quando alguns dos procedimentos requeridos são realizados em estabelecimentos de saúde diversos, há a necessidade de maior deslocamento da usuária, maior disponibilidade de tempo e custeio do transporte quando não garantido pelo poder público.

Diferentes realidades sobre a influência do tipo de serviço sobre a qualidade da atenção prénatal estão descritas em pesquisas científicas. Estudo realizado em município da região Sul do Brasil mostrou que a ESF tem melhor atenção no conjunto das ações relacionadas ao pré-natal, porém as prevalências de realização de alguns dos procedimentos recomendados estão abaixo dos níveis preconizados pelas políticas ${ }^{65}$. Outro estudo realizado na mesma região mostrou que, na ESF, o acesso ao pré-natal é satisfatório, assim como há alta prevalência de realização dos procedimentos clínico-obstétricos indicados para o pré-natal e também maior recebimento de orientações pelas gestantes. Porém, destaca-se que a relação entre os procedimentos clínico-obstétricos e os exames precisa ser melhorada, proporcionando uma continuidade no cuidado ${ }^{47}$. A importância dos Agentes Comunitários de Saúde (ACS) e dos enfermeiros para a adesão e a qualidade do cuidado pré-natal prestado na ESF é reconhecida ${ }^{66}$, também pelas gestantes, que relacionam a atuação das diversas categorias profissionais de saúde e dos ACS ao acesso e continuidade do cuidado pré-natal ${ }^{38}$. Dessa forma, a ESF tem-se mostrado capaz de promover o contato com os serviços de saúde, independente do período gestacional, mas ainda não consegue realizar a captação precoce e a continuidade qualificada do processo de cuidado.

Uma das limitações deste estudo refere-se ao fato de a amostra incluída não ter permitido 
a comparação entre cada um dos municípios da RMGV-ES. Buscou-se contornar essa limitação, incluindo, no desenho metodológico, outras variáveis que complementaram a caracterização do contexto social das mulheres estudadas. Além disso, a exclusão das mulheres que realizaram o pré-natal, pleno ou em parte, no subsistema privado, por um lado, permitiu avançar na análise da política pública para a assistência pré-natal no Brasil, por outro, não permitiu construir uma análise sobre a realidade da totalidade das mulheres que passam pelo período da gravidez.

\section{Conclusões}

Neste estudo observou-se que a alta cobertura da assistência pré-natal não necessariamente vem acompanhada da melhoria da qualidade do cuidado. Além disso, persistem, ainda, desigualdades sociais no acesso ao pré-natal e também geográficas no acesso e na adequação.

A qualidade do cuidado é um processo mais complexo, que requer maior tempo para ser alcançado dentro de um sistema de saúde universal em construção, como o SUS. Também depende de elementos diversos dos necessários para a garantia do acesso, além da oferta de serviços de saúde pré-natal.

A Rede Cegonha, desde 2011, soma-se às diretrizes do PHPN, com o objetivo de estimular a melhoria do acesso, da cobertura e da qualidade do acompanhamento pré-natal, da assistência ao parto e puerpério e da assistência à criança ${ }^{67}$. Para além disso, visando a efetivação da política de atenção materno-infantil, recomenda-se que os gestores incluam a análise da determinação social do desempenho no planejamento e avaliação dos serviços pré-natal e ajustem a alocação de recursos para as áreas com maior necessidade de saúde, permitindo a oferta de cuidados necessários às gestantes, do ponto de vista da integralidade da atenção.

Dada a limitação dos serviços de saúde na redução das desigualdades em saúde, deve-se estabelecer parcerias intersetoriais, promovendo melhores condições de vida e trabalho para que as mulheres e suas comunidades exerçam a liberdade na utilização dos serviços de saúde. Também é preciso qualificar os profissionais para a execução técnica do pré-natal na ESF e para o trabalho interdisciplinar. Isso é imprescindível para a construção de um sistema de saúde equânime, como se propõe a ser o SUS.

Este estudo apresenta alta aplicabilidade para o SUS, para a implementação de políticas que visem à redução de desigualdades dentro da atenção pré-natal, uma vez que apresenta uma nova maneira de se analisar a situação, a partir do Modelo Comportamental da Utilização dos Serviços de Saúde de Andersen ${ }^{6}$.

\section{Colaboradores}

CDD Esposti, C Travassos e RS Pinheiro participaram de todas as etapas da pesquisa, incluindo a concepção, análise e interpretação dos dados, redação e revisão do artigo e aprovação da versão a ser publicada; ET Santos Neto participou da concepção, análise e interpretação dos resultados, revisão crítica e aprovação da versão a ser publicada; e AE Oliveira colaborou na interpretação dos dados, revisão crítica do artigo e aprovação da versão a ser publicada. 


\section{Agradecimento}

À Coordenação de Aperfeiçoamento de Pessoal de Nível Superior (Capes), pelo pagamento de bolsa de doutorado para a primeira autora.

\section{Referências}

1. Rawls J. Justiça como equidade: uma concepção política, não metafísica. Lua Nova 1992; (25):28-59.

2. Rawls J. Uma teoria da Justiça. São Paulo: Martins Fontes; 1997.

3. Sen A. Why equity health? Health Econ. 2002; 11:659666.

4. Sen A. Why and how is health a human right? Lancet 2008; 372(9655):2010.

5. Organização Mundial da Saúde (OMS). A conceptual framework for action on the social determinants of health: Social Determinants of Health Discussion. Geneve: OMS; 2010. Paper 2 (Policy and Practice).

6. Andersen RM. National Health Surveys and the Behavioral Model of Health Services Use. Med. Care 2008; 46(7):647-653.

7. Viacava F, Viacava F, Ugá MAD, Porto S, Laguardia J, Moreira RS. Avaliação de Desempenho de Sistemas de Saúde: um modelo de análise. Cien Saude Colet 2012; 17(4):921-934.

8. Mooney GH. Equity in health care: confronting the confusion. Eff. Health Care 1983; 1(4):179-185.

9. Paim J, Travassos C, Almeida C, Bahia L, Macinko J. The Brazilian health system: history, advances, and challenges. Lancet 2011; 377(9779):1778-1797.

10. Arsenault C, Jordan K, Lee D, Dinsa G, Manzi F, Marchant T, Kruk ME. Equity in antenatal care quality: an analysis of 91 national household surveys. Lancet Glob Health 2018; 6(11):e1186-e1195.

11. Victora CG, Aquino EML, Leal MC, Monteiro CA, Barros FC, Szwarcwald CL. Saúde de mães e crianças no Brasil: progressos e desafios. Lancet 2011 [acessado 2019 maio 30]; 32-46. Disponível em: http://bvsms. saude.gov.br/bvs/artigos/artigo_saude_brasil_2.pdf.

12. Viellas EF, Domingues RMSM, Dias MAB, Gama SGN, Theme Filha MM, Costa JV, Bastos HM, Leal MC. Assistência pré-natal no Brasil. Cad Saude Publica [Internet]. 2014 [cited 2019 May 30]; 30(Supl. 1):S85-S100. Available from: http://www.scielo.br/ scielo.php?script=sci_arttext\&pid=S0102-311X2014 001300016\&lng=en

13. Andreucci CB, Cecatti JG. Desempenho de indicadores de processo do Programa de Humanização do Pré-natal e Nascimento no Brasil: uma revisão sistemática. Cad Saude Publica 2011; 27(6):1053-1064.

14. Gonçalves CV, Cesar JA, Mendonza-Sassi RAM. Qualidade e equidade na assistência à gestante: um estudo de base populacional no Sul do Brasil. Cad Saude Publica 2009; 25(11)2507:2516.

15. Ribeiro ERO, Guimarães AMDN, Bettiol H, Lima DDF, Almeida MLD, Souza L, Silva AAM, Gurgel RQ. Risk factors for inadequate prenatal care use in the metropolitan area of Aracaju, Northeast Brazil. BMC Pregnancy and Childbirth 2009; 9:31.

16. Almeida SDM, Barros MBA. Equidade e atenção à saúde da gestante em Campinas (SP), Brasil. Rev. Panam. Salud Pública 2005; 17(1):15-25.

17. Coimbra LC, Silva AAM, Mochel EG, Alves MTSSB, Ribeiro VS, Aragão VMF, Bettiol H. Fatores associados à inadequação do uso da assistência pré-natal. Rev Saude Publica 2003; 37(4):456-462. 
18. Brasil. Ministério da Saúde (MS). Departamento de Ciência e Tecnologia, Secretaria de Ciência, Tecnologia e Insumos Estratégicos. Avaliação Nacional do Programa de Humanização do Pré-natal e Nascimento. Rev Saude Publica 2008; 42(2):383-387.

19. Fonsecal CRB, Strufaldi MWL, Carvalho LR, Puccini RF. Adequacy of antenatal care and its relashionship with low birth weight in Botucatu, São Paulo, Brazil: a case control study. BMC Pregnancy and Childbirth 2014; 14:255-267.

20. Lansky S, Friche AAL, Silva AAM, Campos D, Bittencourt DAS, Carvalho ML, Frias PG, Cavalcante RS, Cunha AJLA. Pesquisa Nascer no Brasil: perfil de mortalidade neonatal e avaliação da assistência à gestante e ao recém-nascido. Cad Saude Publica 2014; 30(Supl.)S192:S207.

21. Barros FC, Bhutta ZA, Batra M, Hansen TN, Victora CG, Rubens CE. Global report on preterm birth and stillbirth ( 3 of 7): evidence for effectiveness of interventions. BMC Pregnancy and Childbirth 2010; 10(Supl. 1):S3-39.

22. Barros FC, Matijasevich A, Requejo JH, Giugliani E, Maranhão AG, Monteiro CA, Barros AJD, Bustreo F, Merialdi M, Victora CG. Recent trends in maternal, newborn, and child health in Brazil: progress toward Millennium Development Goals 4 and 5. Am J Public Health 2010; 100(10):1877-1889.

23. Silveira MF, Victora CG, Barros AJD, Santos IS, Matijasevich A, Barros FC. Determinants of preterm birth: Pelotas, Rio Grande do Sul State, Brazil, 2004 birth cohort. Cad Saude Publica 2010; 26(1):185-194.

24. Brasil. Ministério da Saúde (MS). Portaria GM/MS $\mathrm{n}^{\circ}$ 569, de 1 junho de 2000. Diário Oficial da União 2000; 1 jun.

25. Victora CG, Hanson K, Bryce J, Vaughan PJ. Achieving universal coverage with health interventions. Lancet 2004; 364(9444):1541-1548.

26. Instituto Brasileiro de Geografia e Estatística (IBGE). Conheça cidades e estados do Brasil. [acessado $2019 \mathrm{Fev}$ 05]. Disponível em: https://cidades.ibge.gov.br/

27. Instituto Jones Dos Santos Neves (IJSN). Produto Interno Bruto dos Municípios - 2016. Vitória: IJSN; 2018.

28. Santos-Neto ET, Oliveira, AE, Zandonade E, Gama SGN, Leal MC. O que os cartões de pré-natal das gestantes revelam sobre a assistência nos serviços do SUS da Região Metropolitana da Grande Vitória, Espírito Santo, Brasil? Cad Saude Publica 2012; 28(11):16501662.

29. Polgliane RBS, Leal MC, Amorim MHC, Zandonade E, Santos Neto ET. Adequação do processo de assistência pré-natal segundo critérios do Programa de Humanização do Pré-natal e Nascimento e da Organização Mundial de Saúde. Cien Saude Colet 2014; 19(7):1999-2010.

30. Atlas Brasil. Atlas Brasil 2013. [acessado 2014 Jun 18]. Disponível em: http://www.atlasbrasil.org.br/2013/

31. World Health Organization (WHO). The World Health Organization's infant-feeding recommendation. Bull. World Health Organization 1995; 73:165174 .
32. Swarcwald CL, Leal MC, Gouveia GC, Souza WV. Desigualdades socioeconômicas em saúde no Brasil: resultados da Pesquisa Mundial de Saúde, 2003. Rev. Bras. Saúde Matern. Infant. 2005; 5(Supl. 1):S11-S22.

33. Vettore MV, Gama SGN, Lamarca GA, Schilithz AOC, Leal MC. Housing conditions as a social determinant of low birthweight and preterm low birthweight. Rev Saude Publica 2010; 44(6):1021-1031.

34. Thiede M, Akewengo P, Mc'Intyre D. Explorando as dimensões do acesso. In: Mc'Intyre D, Mooney G. Aspectos econômicos da equidade em saúde. Rio de Janeiro: Editora Fiocruz; 2014. p. 137-161.

35. Saavedra JS, Cesar, JA. Uso de diferentes critérios para avaliação da inadequação do pré-natal: um estudo de base populacional no extremo Sul do Brasil. Cad Saude Publica 2015; 31(5):1003-1014.

36. Mallmann MB, Boing AF, Tomasi YT, Anjos JC, Boing AC. Evolução das desigualdades socioeconômicas na realização de consultas de pré-natal entre parturientes brasileiras: análise do período 2000-2015. Epidemiol. Serv. Saude 2018; 27(4):7-11.

37. Ribeiro JM, Costa NR, Pinto LFS, Silva PLB. Atenção ao pré-natal na percepção das usuárias do SUS. Cad Saude Publica 2004; 20(2):534-545.

38. Esposti CDD, Oliveira AE, Santos-Neto ET, Travassos CMR. Representações sociais sobre o acesso e o cuidado pré-natal no Sistema Único de Saúde da Região Metropolitana da Grande Vitória, Espírito Santo. Saúde Soc. 2015; 24(3):765-779.

39. Figueiredo PP, Rossoni E. O acesso à assistência pré-natal na Atenção Básica à Saúde sob a ótica das gestantes. Rev. Gaúcha Enferm. 2008; 29(2):238-245.

40. Almeida CAL, Tanaka OY. Perspectiva das mulheres na avaliação do Programa de Humanização do Pré-Natal e Nascimento. Rev Saude Publica 2009; 43(1):98-104.

41. Albuquerque RA, Jorge MSB, Franco TB, Quinderé PHD. Produção do cuidado integral no pré-natal: itinerário de uma gestante em uma unidade básica de saúde da família. Interface (Botucatu) 2011; 15(38):677-686.

42. Vieira SM, Bock LF, Zocche DA, Pessota CU. Percepção das puérperas sobre a assistência prestada pela equipe de saúde no pré-natal. Texto \& Contexto Enferm. 2011; 20(n. esp.):255-262.

43. Coutinho T, Teixeira MTB, Dain S, Sayd JD, Coutinho LM. Adequação do processo de assistência pré-natal entre as usuárias do Sistema Único de Saúde em Juiz de Fora-MG. Rev. Bras. Ginecol. Obstet. 2003; 25(10):717-724.

44. Coutinho T, Monteiro MFG, Sayd JD, Teixeira MTB, Coutinho CM, Coutinho LM. Monitoramento do processo de assistência pré-natal entre as usuárias do Sistema Único de Saúde em município do Sudeste brasileiro. Rev. Bras. Ginecol. Obstet. 2010; 32(11):563569.

45. Moraes MS, Kujumjian FG, Chiaravalloti Neto F, Lopes JCC. Avaliação da assistência às gestantes: o caso do município de São José do Rio Preto, São Paulo, Brasil. Rev. Bras. Saúde Mater. Infant. 2004; 4(4):375-384. 
46. Koffman MD, Bonadio IC. Avaliação da atenção pré-natal em uma instituição filantrópica da cidade de São Paulo. Rev. Bras. Saúde Mater. Infant. 2005; 5(Supl. 1):s23-32.

47. Anversa ETR, Bastos GAN, Nunes LN, Dal Pizzol TS. Qualidade do processo da assistência pré-natal: unidades básicas de saúde e unidades de Estratégia Saúde da Família em município no Sul do Brasil. Cad Saude Publica 2012; 28(4):789-800.

48. Domingues RMSM, Hartz ZMA, Dias MAB, Leal MC. Avaliação da adequação da assistência pré-natal na rede SUS do Município do Rio de Janeiro, Brasil. Cad Saude Publica 2012; 28(3):425-437.

49. Martinelli KG, Santos Neto ET, Gama SGN, Oliveira AE. Adequação do processo da assistência pré-natal segundo os critérios do Programa de Humanização do Pré-natal e Nascimento (PHPN) e Rede Cegonha. Rev. Bras. Ginecol. Obstet. 2014; 36(2):56-64.

50. Silva EP, Lima RT, Osório MM. Impacto de estratégias educacionais no pré-natal de baixo risco: revisão sistemática de ensaios clínicos randomizados. Cien Saude Colet 2016; 21(9):2935-2948.

51. Souza VB, Roecker S, Marcon SS. Ações educativas durante a assistência pré-natal: percepção de gestantes atendidas na rede básica de Maringá-PR. Rev. Eletr. Enf. [Internet], 2011 [acessado 2015 Mar 17]; 13(2):199-210. Disponível em: http://www.fen.ufg.br/ revista/v13/n2/v13n2a06.htm.

52. Mendoza-Sassi RA, Cesar JA, Ulmi EF, Mano PS, Dall'Agnol MM, Neumann NA. Avaliando o conhecimento sobre pré-natal e situações de risco à gravidez entre gestantes residentes na periferia da cidade de Rio Grande, Rio Grande do Sul, Brasil. Cad Saude Publica 2007; 23(9):2157-2166.

53. Figueroa V, Alfaro NA, Rosa AC. Predictores de utilización de la atención prenatal. Aten. Prim. 2005; 35(4):185-191.

54. Say L, Raine R. A systematic review of inequalities in the use of maternal health care in developing countries: examining the scale of the problem and the importance of context. Bulletin of the World Health Organization 2007; 85(10):812-819.

55. Puccini RF. Eqüidade na atenção pré-natal e ao parto em área da Região Metropolitana de São Paulo, 1996. Cad Saude Publica 2003; 19(1):35-45.

56. Domingues RMSM, Leal MC, Hartz ZMA, Dias MAB, Vettore MV. Acesso e utilização de serviços de pré-natal na rede SUS do município do Rio de Janeiro, Brasil. Rev Bras Epidemiol 2013; 16(4):953-965.

57. Rasia ICRB, Albernaz E. Atenção pré-natal na cidade de Pelotas, Rio Grande do Sul, Brasil. Rev. Bras. Saúde Matern. Infant. 2008; 8(4):401-410.

58. Jong EIF. Determinants of late and/or inadequate use of prenatal healthcare in high-income countries: a systematic review. Eur J Public Health 2015; 22(6):904-913.

59. Barreto SM, Passos VMA, Giatti L. Comportamento saudável entre adultos jovens no Brasil. Rev Saude Publica 2009; 43(Supl. 2):9-17.
60. Fonseca SC, Kale PL, Silva KS. Pré-natal em mulheres usuárias do Sistema Único de Saúde em duas maternidades no Estado do Rio de Janeiro, Brasil: a cor importa? Rev. Bras. Saúde Matern. Infant. 2015; 15(2):209-217.

61. Barreira TMHM, Makhoul AF, Cohn A. Condição feminina de mulheres chefes de família em situação de vulnerabilidade social. Serv. Soc. Soc. 2011; (105):167179.

62. Espírito Santo. Ficha de informações municipais de 2013. [acessado 2015 Fev 26]. Disponível em: http:// www.saude.es.gov.br/default.asp

63. Brasil. Ministério da saúde (MS). Índice de Desempenho do Sistema Único de Saúde: Resultados. Brasília; 2011. [acessado 2015 Mar 01]. Disponível em: http:// idsus.saude.gov.br/mapas.html

64. Porto SM. Equidade na distribuição geográfica dos recursos em saúde: uma contribuição para o caso brasileiro [tese]. Fundação Oswaldo Cruz: Rio de Janeiro; 1997.

65. Mendoza-Sassi RAM. Diferenças no processo de atenção ao pré-natal entre unidades da Estratégia Saúde da Família e unidades tradicionais em um município da Região Sul do Brasil. Cad Saude Publica 2011; 27(4):787-796

66. Duarte SJH, Andrade SM. O significado do pré-natal para mulheres grávidas: Uma experiência no município de Campo Grande, Brasil. Saúde Soc 2008; 17(2):132-139.

67. Brasil. Ministério da Saúde (MS). Portaria GM/GM $\mathrm{n}^{\circ}$ 2.351, de 5 de outubro de 2011. Institui, no âmbito do Sistema Único de Saúde - SUS - a Rede Cegonha. Diário Oficial da União 2011; 5 out.

Artigo apresentado em 30/05/2019

Aprovado em 07/08/2019

Versão final apresentada em 11/11/2019 
